\title{
Fault identification of gearbox degradation with optimized wavelet neural network
}

\author{
Hanxin Chen*, Yanjun Lu and Ling Tu \\ School of Mechanical and Electrical Engineering, Wuhan Institute of Technology, Wuhan, Hubei, China
}

Received 7 April 2012

Revised 11 June 2012

\begin{abstract}
A novel intelligent method based on wavelet neural network (WNN) was proposed to identify the gear crack degradation in gearbox in this paper. The wavelet packet analysis (WPA) is applied to extract the fault feature of the vibration signal, which is collected by two acceleration sensors mounted on the gearbox along the vertical and horizontal direction. The backpropagation (BP) algorithm is studied and applied to optimize the scale and translation parameters of the Morlet wavelet function, the weight coefficients, threshold values in WNN structure. Four different gear crack damage levels under three different loads and three various motor speeds are presented to obtain the different gear fault modes and gear crack degradation in the experimental system. The results show the feasibility and effectiveness of the proposed method by the identification and classification of the four gear modes and degradation.
\end{abstract}

Keywords: Fault identification, wavelet neural network, back-propagation algorithm, gearbox degradation

\section{Introduction}

Gearbox is one of the most important components to transmit power and torque effectively from one shaft to another in mechanical system. It is typical of the applications in aircrafts, navigations and automotive industries. The working condition of the gearbox has the great influence on the function and performance of the mechanical equipment. When the critical components of the machine fail during the operation, the unexpected downtime causes tremendous economic loss and disastrous accident. It is significant to do research on fault diagnosis of the gearbox. In the past decades, a large number of investigations on fault diagnosis of gearbox have been done [1]. However, it is still necessary to improve the speed and accuracy of diagnosis of certain machinery components. There are a lot of theories and methods about fault diagnosis and condition monitoring of the rotating machinery based on artificial intelligence techniques [2,3].

With the development of modern high science and technology, many automated and intelligent methods had been used to diagnose faulty gears in gearbox in recent years. An intelligent method of fault diagnosis based on artificial neural network (ANN) has been used in condition monitoring and fault diagnosis of gearbox [4]. It increases the reliability of fault diagnosis of gear in the gearbox testing system. Zhang [5] first proposed the concept of wavelet neural network (WNN) that is a feed-forward neural network based on wavelet transform, which is a very effective method in identifying and classifying for non-stationary signals. Several new models of WNN were proposed and developed in Refs [6,7]. WNN has been widely applied in fault diagnosis and prognosis due to its unique capabilities, such as nonlinear function approximation, pattern recognition and classification [8]. WNN has been successfully applied to fault diagnosis and detection of gears to achieve very good effects. Kang adopted WNN to recognize and classify accurately for several gear fault types, for instance, tooth breakage and wear [9]. WNN was used to identify

\footnotetext{
* Corresponding author: Hanxin Chen, School of Mechanical and Electrical Engineering, Wuhan Institute of Technology, Wuhan, Hubei, China. E-mail: pg01074075@163.com.
} 
and detect the abrasion faults on the different gears in gearbox [10]. The gear faults have a large proportion in all kinds of fault categories of gearbox. The gear crack is a common form of gear faults. It is important and necessary to further explore identification and classification abilities of WNN and achieve better performance with the higher diagnostic accuracy.

It is great challenge to develop the method to diagnose the gear cracks in gearbox automatically and accurately. In Ref [11], the authors applied the weighted K nearest neighbor classification algorithm for the fault diagnosis of the gear cracks. However, there is no intelligent method to assess the value of the coefficient $\mathrm{K}$ and feature weighting, which have great effect on the results of fault diagnosis. A multidimensional hybrid intelligent method for gear fault diagnosis was proposed in [12]. This method is very complicated and takes more time to get the final classification results. It is still necessary for both of the above methods to improve the classification results of the fault diagnosis as well as the number of the types of the gear crack levels. Compared with the two above methods, the robust and simple intelligent method in this paper is proposed to identify the more gear crack levels under the separate operating conditions with the different loads and rotational speeds.

Wavelet packet analysis (WPA) is a typical signal processing method, which is very suitable for non-stationary vibration signal analysis. Fault characteristic information of vibration signals from mechanical system has been extracted successfully by using WPA [13]. In this paper, the research on fault diagnosis of the different damage levels under the crack fault category has been done. The method based on WNN is studied to identify the gear fault modes and damage levels under three different loads and rotational speeds of gearbox. The comparison on the capability of identifying the fault modes of the gear crack in gearbox between the proposed method in this paper and the methods in Refs [11,12] is analyzed, which shows the advantages of the proposed method over the others in Refs $[11,12]$.

\section{Feature extraction with wavelet packet analysis (WPA)}

The feature extraction of the relevant characteristic information from the vibration signals is important technique for the identification and classification of the multi-fault mode in the fault diagnosis of engineering system. WPA decomposes the original signal to be the low and high frequency signal simultaneously and overcomes the disadvantage of multi-resolution analysis, which does not make a further decomposition for high frequency parts of signal. WPA adaptively selects the appropriate frequency band to match the original signal with the signal spectrum based on the characteristics of analyzed signal, which improves the time-frequency resolution. WPA is the advanced signal analysis and used to obtain the feature values of vibration signals, which is inputted to WNN as the feature vectors. The definition of a wavelet packet function $W_{j, k}^{n}(t)$ is as follows [14]:

$$
W_{j, k}^{n}(t)=2^{j / 2} W^{n}\left(2^{j} t-k\right)
$$

Where $n=0,1,2, \ldots$ is the oscillation parameter, $j \in Z$ and $k \in Z$ are the scaling parameter and the translation parameter. When $n=0,1 ; j=k=0$, the two wavelet packet functions equal to the scaling function $\phi(t)$ and the mother wavelet function $\psi(t)$.

$$
\begin{aligned}
& W^{0}(t)=\phi(t) \\
& W^{1}(t)=\psi(t)
\end{aligned}
$$

The recursive relationship defines the other wavelet packet functions for $n=2,3, \ldots$ by the low-pass filter $h(k)$ and the high-pass filter $g(k)[14]$.

$$
\begin{aligned}
& W^{2 n}(t)=\sqrt{2} \sum_{k \in z} h(k) W^{n}(2 t-k) \\
& W^{2 n+1}(t)=\sqrt{2} \sum_{k \in z} g(k) W^{n}(2 t-k)
\end{aligned}
$$




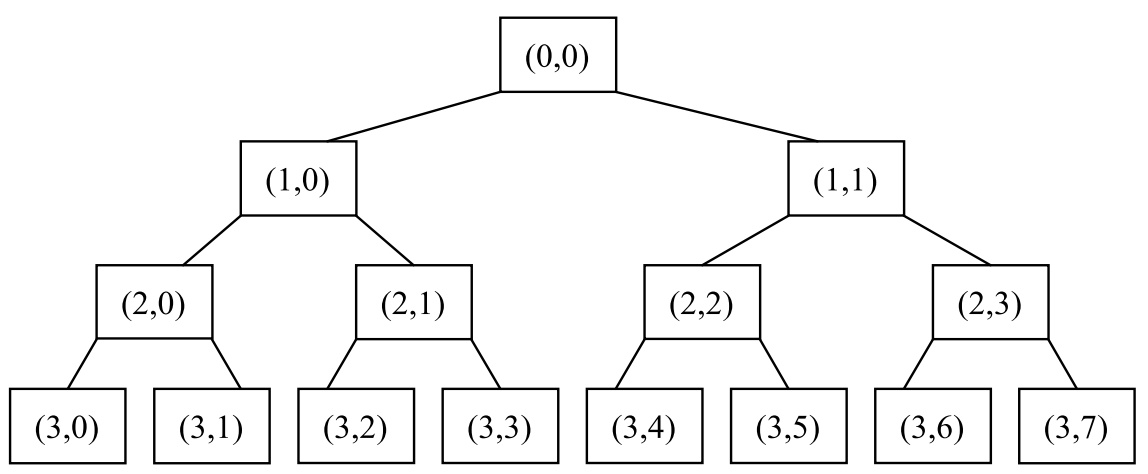

Fig. 1. The binary tree structure of three layer wavelet packet decomposition.

$$
\begin{aligned}
& h(k)=1 / \sqrt{2}\langle\phi(t), \phi(2 t-k)\rangle \\
& g(k)=1 / \sqrt{2}\langle\psi(t), \psi(2 t-k)\rangle=(-1)^{k} h(1-k)
\end{aligned}
$$

Here, $\langle\cdot, \cdot\rangle$ stands for the inner product operator.

The WPA coefficients for a signal $x(t)$ are calculated by the low-pass and high-pass filters iteratively. Let $d_{j, k}^{n}$ denote the decomposition coefficients [15]:

$$
d_{j, k}^{n}=\left\langle x, W_{j, k}^{n}\right\rangle=\int_{-\infty}^{+\infty} x(t) W_{j, k}^{n}(t) d t
$$

The coefficient $d_{j, k}^{n}$ corresponds the specific frequency band, which is presented by the node $(j, n)$. By calculating the full wavelet packet decomposition of a discrete time signal $x(t), 2^{j}$ sets of WPA coefficients at $j$ level are obtained. Here $j$ is the decomposition level and $n$ is the order of the node position in the specific level. For a discrete signal $x(t)=\left\{d_{0}^{1}(k) \mid k=1,2, \ldots, N\right\}, N$ is the length of the signal. The decomposition coefficients of wavelet packet are calculated as [14]:

$$
\begin{aligned}
& d_{j+1}^{2 n}=\sum_{k} h(k-2 t) d_{j}^{n}(k) \\
& d_{j}^{2 n+1}=\sum_{k} g(k-2 t) d_{j}^{n}(k)
\end{aligned}
$$

The reconstructed coefficient of wavelet packet is as follows [14]:

$$
d_{j}^{n}(k)=2\left[\sum_{\tau} h(k-2 \tau) d_{j+1}^{2 n+1}(k)+\sum_{\tau} g(k-2 \tau) d_{j+1}^{2 n}(k)\right]
$$

Where $d_{j}^{n}(k)$ denotes the $k$ th reconstructed coefficient of wavelet packet tree at node $(j, n), j=3$ and $n=$ $1,2, \ldots, 2^{j}$. The original signal $x(t)$ is obtained through the following two equations:

$$
\begin{aligned}
& x(t)=\sum_{n=1}^{2^{j}} x_{j}^{n}(t) \\
& x_{j}^{n}(t)=\sum_{k=1}^{N} d_{j, k}^{n} W_{j, k}^{n}(t)
\end{aligned}
$$


Table 1

The input feature parameters in WNN

\begin{tabular}{lllllllll}
\hline Feature parameter & $E_{1}$ & $E_{2}$ & $E_{3}$ & $E_{4}$ & $E_{5}$ & $E_{6}$ & $E_{7}$ & $E_{8}$ \\
\hline Identifier & $x_{1}$ & $x_{2}$ & $x_{3}$ & $x_{4}$ & $x_{5}$ & $x_{6}$ & $x_{7}$ & $x_{8}$ \\
\hline
\end{tabular}

The original signal is decomposed by wavelet packet, the $2^{j}$ orthogonal frequency bands from low frequency to high frequency at $\mathrm{j}_{\text {th }}$ level are obtained, that is the energy of the original vibration signal is decomposed into $2^{j}$ different orthogonal frequency bands. The sum of energies of signals in all frequency bands equals to the energy of original signal, and the vibration signal in each band represents the characteristics information of the original signal in the corresponding frequency range. The binary tree structure of three layer wavelet packet decomposition is shown in Fig. 1. The procedures that extract the feature parameters of vibration signal by wavelet packet decomposition as follows:

(1) Use the three-level decomposition of WPA to analyze the original vibration signal from the gearbox and generate eight subbands. The Haar wavelet is chosen as the wavelet packet basis function [3] and Shannon entropy is selected as the entropy standard to analyze the original vibration signal, which has no relation with the following Morlet wavelet function in WNN.

(2) Calculate the energy value of feature signal in each frequency band. $\left(S_{1}, S_{2}, \ldots, S_{8}\right)$ denotes the signals from the low frequency subband to the high frequency subband. The original signal is $S=S_{1}+S_{2}+\cdots+S_{8}$. The wavelet packet node energy $E_{n}$ of the signal $S_{n}(n=1,2, \ldots, 8)$ is defined as:

$E_{3 n}=\int S_{3 n}(t)^{2} d t=\sum_{k=1}^{N}\left|d_{j}^{n}(k)\right|^{2}$

(3) Construct a set of feature vector. There are eight energy values from low frequency to high frequency in three-layer wavelet packet decomposition. The feature vector $T=\left[E_{1}, E_{2}, E_{3}, E_{4}, E_{5}, E_{6}, E_{7}, E_{8}\right]$ consists of the eight energy values, which are used as a set of feature parameters of WNN and shown in Table 1.

(4) Normalize a set of feature vector $X=\left[x_{1}, x_{2}, \ldots, x_{8}\right]$ by using the following equations, a new vector $X^{\prime}$ as feature input vector of WNN is obtained.

$$
\begin{aligned}
\bar{X} & =\left(\sum_{n=1}^{8}\left|x_{n}\right|^{2}\right)^{1 / 2} \\
X^{\prime} & =\left[x_{1} / \bar{X}, x_{2} / \bar{X}, \ldots, x_{8} / \bar{X}\right]
\end{aligned}
$$

\section{The theory and algorithm on wavelet neural network}

Wavelet neural network (WNN) has attracted much attention in signal processing and pattern recognition [16]. WNN has combined the advantages of wavelet analysis and neural network, which owns not only the wavelet's time frequency localization ability but also the neural network's capacity to be self-learning and self-adaptive so that it has stronger capabilities of function approximation and fault tolerance than the conventional ANN in dealing with nonlinear mapping and signal classification. The basic idea of WNN is employing non-orthogonal wavelet function as the activation function of hidden layer instead of Sigmoid activation function. The wavelet function is able to be controlled and adjusted to solve specific problems according to different practical applications by the operation of the dilation and translation. The wavelet basis function in WNN structure can detect the characteristics of local and global from the raw signal simultaneously in both time and frequency domain. WNN is very well suitable for solving non-stationary signal processing, non-linear system identifying and pattern recognition.

The wavelet transform uses a series of oscillating functions with different frequencies as window function to analyze the signal. The fundamental principle of wavelet transform is to approximate a signal by the construction of a set of specific wavelet basis functions. Compared with the traditional Fourier transform that only analyses signal in time domain or frequency domain, wavelet transform analyzes the signal in time-frequency domain. The wavelet 


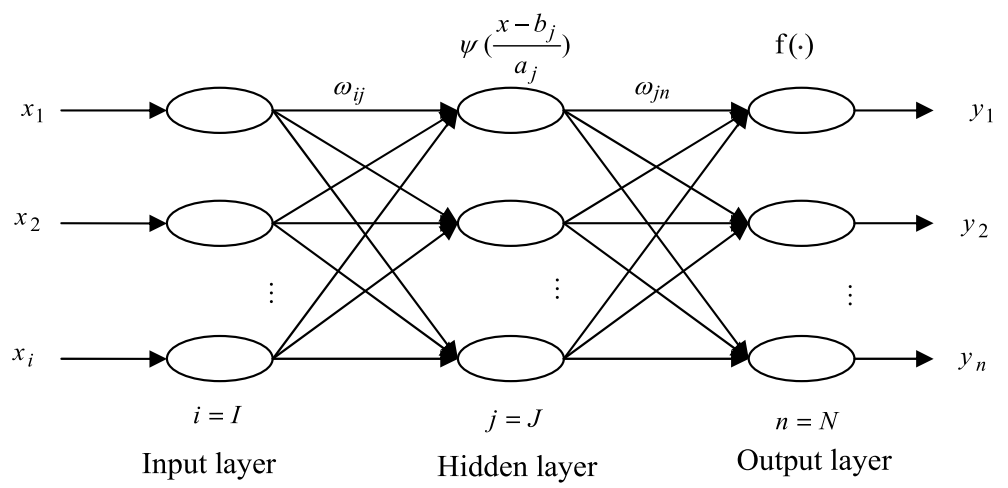

Fig. 2. The three-layer architecture of the wavelet neural network.

function is characteristic of the superior localization performance and is more suitable for the multi-resolution analysis of the non-stationary signal in both time and frequency domain through the operation of the dilation and translation. If a mother wavelet is defined as $\psi(x) \in L^{2}(R) . L^{2}(R)$ denotes the square integrate real space. Ris the set of real numbers. The daughter wavelet function is the following form:

$$
\psi_{a, b}(x)=\frac{1}{\sqrt{a}} \psi\left(\frac{x-b}{a}\right) \quad(a, b \in R, a>0)
$$

Where $a, b$ are the dilation and translation parameter. The continuous wavelet transform of the signal $f(x) \in$ $L^{2}(R)$ is defined as:

$$
W_{f}(a, b)=\int_{0}^{+\infty} \psi_{a, b}^{*}(x) f(x) d x=\frac{1}{\sqrt{a}} \int_{0}^{+\infty} \psi^{*}\left(\frac{x-b}{a}\right) d x
$$

Where $W_{f}(a, b)$ is wavelet transform coefficient and $\psi_{a, b}^{*}(x)$ is the complex conjugate of $\psi_{a, b}(x)$. The original signal is reconstructed by inverse transformation defined as:

$$
f(x)=\frac{1}{C_{\psi}} \int_{0}^{+\infty} \frac{d a}{a^{2}} \int_{-\infty}^{+\infty} W_{f}(a, b) \psi_{a, b}(x) d b
$$

Wavelet neural network (WNN) is a three-layer network structure with an input layer, a hidden layer and an output layer. It employs the nonlinear wavelet basis function as the activation function, instead of the common Sigmoid function in hidden layer. It has the advantages of both wavelet analysis and neural network, and has been widely applied in many fields, such as pattern recognition, signal processing, fault diagnosis etc. In previous researches, most researchers adopted the Morlet wavelet as the wavelet function of the hidden layer of WNN. So the Morlet wavelet in Eq. (20) is selected as the wavelet of the hidden layer and the Sigmoid function in Eq. (21) is used as the activation function of the output layer. The model of WNN is shown in Fig. 2.

$$
\begin{aligned}
& \psi\left(\frac{x-b}{a}\right)=\cos \left(1.75\left(\frac{x-b}{a}\right)\right) \exp \left(-0.5\left(\frac{x-b}{a}\right)^{2}\right) \\
& f(\cdot)=1 / 1+\exp (-x)
\end{aligned}
$$

As illustrated in Fig. 2, the input vector $X^{\prime}$ of input layer of the network is expressed as $x=\left[x_{1}, x_{2}, \ldots, x_{i}\right]$, which is the extracted feature vector from the original vibration signal. The vector $y=\left[y_{1}, y_{2}, \ldots, y_{n}\right]$ is the output of the output layer. The parameters $I, J, N$ are the nodes number of the input layer, the hidden layer and the output 
layer, respectively. The parameters $\omega_{i j}$ and $\omega_{j n}$ are the connecting weights between the input layer and the hidden layer, and between the hidden layer and the output layer. The mapping relationship of the WNN network from the parameter $x_{i}^{p}$ of the input layer to the parameter $y_{n}^{p}$ of the output layer is defined as follows [5]:

$$
I_{j}^{p}=\sum_{i=1}^{I} \omega_{i j} x_{i}^{p}+b 1_{j}
$$

Where the parameter $p$ is the sample number, the parameter $\omega_{i j}$ is the connecting weight between the $i_{\text {th }}$ unit of the input layer and the $\mathrm{j}_{\mathrm{th}}$ unit of the hidden layer, the parameter $x_{i}^{p}$ denotes the input of the $\mathrm{i}_{\mathrm{th}}$ node of the $\mathrm{p}_{\mathrm{th}}$ sample in the input layer, the parameter $b 1_{j}$ is the threshold value of the $j_{t h}$ node of the hidden layer, the parameter $I_{j}^{p}$ is the input of the $\mathrm{j}_{\mathrm{th}}$ node of the $\mathrm{p}_{\mathrm{th}}$ sample in the hidden layer.

$$
O_{j}^{p}=\psi\left(\frac{I_{j}^{p}-b_{j}}{a_{j}}\right)
$$

The parameter $O_{j}^{p}$ is the output of the $j_{\text {th }}$ node of the $\mathrm{p}_{\text {th }}$ sample in the hidden layer, the parameters $a_{j}$ and $b_{j}$ are the scale and translation coefficients of the Morlet wavelet function at the $\mathrm{j}_{\mathrm{th}}$ node in the hidden layer. The Morlet wavelet function in Eq. (20) is used in Eq. (23) to obtain the following equation.

$$
I_{n}^{p}=\sum_{j=1}^{J} \omega_{j n} O_{j}^{p}
$$

The parameter $\omega_{j n}$ is the connecting weight from the $j$ th unit of the hidden layer to the $n$th unit of the output level, the parameter $I_{n}^{p}$ is the input of the $n$th node of the $\mathrm{p}_{\text {th }}$ sample in the output layer.

$$
y_{n}^{p}=f\left(I_{n}^{p}+b 2_{n}\right)
$$

The parameter $y_{n}^{p}$ is the output of the $n$th node of the $p$ th sample in the output layer, the parameter $b 2_{n}$ is the threshold value of the $n$th node of the output layer. The right of Eq. (25) is the Sigmoid activation function in the output layer, which is shown in Eq. (21).

The output function of WNN is described as the following equation [5]:

$$
y_{n}^{p}=f\left[\sum_{j=1}^{J} \omega_{j n} \psi\left(\left(\left(\sum_{i=1}^{I} \omega_{i j} x_{i}^{p}+b 1_{j}\right)-b_{j}\right) / a_{j}\right)+b 2_{n}\right]
$$

The traditional back-propagation algorithm that is the gradient descent algorithm is applied to optimize the parameters of WNN. The parameters of the network are optimized by the minimization of the following objective error function in the continuous learning and training procedure:

$$
E=\sum_{p=1}^{P} E^{p}=\frac{1}{2} \sum_{p=1}^{P} \sum_{n=1}^{N}\left(d_{n}^{p}-y_{n}^{p}\right)^{2}
$$

Where the parameter $d_{n}^{p}$ is the expected output of $n$th node in output layer, the parameter $y_{n}^{p}$ is the actual output of the network.

During the procedure of the calculation of the WNN parameters from the input $x_{i}^{p}$ to the output $y_{n}^{p}$ in Eqs (22)(25), the back-propagation error value is calculated until the output error $E$ meets the requirement, which is shown as follows [5]:

$$
\delta_{n}=-\frac{\partial E}{\partial I_{n}^{p}}=\left(d_{n}^{p}-y_{n}^{p}\right) y_{n}^{p}\left(1-y_{n}^{p}\right)
$$




$$
\delta_{j}=-\frac{\partial E}{\partial I_{j}^{p}}=\sum_{n=1}^{N} \delta_{n} \omega_{j n} \frac{\partial O_{j}^{p}}{\partial I_{j}^{p}}=\sum_{n=1}^{N} \delta_{n} \omega_{j n} \psi^{\prime}\left(\left(I_{j}^{p}-b_{j}\right) / a_{j}\right) \frac{1}{a_{j}}
$$

Where parameter $\delta_{n}$ is the back-propagation error value of the output layer and the parameter $\delta_{j}$ is the backpropagation error value of the hidden layer. The parameter $\psi^{\prime}(\cdot)$ is the differentiation equation of the parameter $\psi(\cdot)$.

The learning rate is $\eta$ and the momentum term is $\lambda$. All variables of the network are calculated by using the error values $\delta_{n}$ and $\delta_{j}$ in BP algorithm. The variables $\Delta \omega_{i j}$ and $\Delta \omega_{j n}$ used in the following Eqs (36) and (37) are defined as follows [5]:

$$
\begin{aligned}
& \Delta \omega_{i j}=-\eta \frac{\partial E}{\partial \omega_{i j}}=\eta \delta_{n} \omega_{j n} \frac{\partial O_{j}^{p}}{\partial I_{j}^{p}} x_{i}^{p}=\eta \delta_{j} x_{i}^{p} \\
& \Delta \omega_{j n}=-\eta \frac{\partial E}{\partial \omega_{j n}}=\eta \delta_{n} O_{j}^{p}
\end{aligned}
$$

The wavelet parameters $\Delta a_{j}$ and $\Delta b_{j}$ used in following Eqs (38) and (39) are defined as follows:

$$
\begin{aligned}
& \Delta a_{j}=-\eta \frac{\partial E}{\partial a_{j}}=\eta \delta_{n} \omega_{j n} \frac{\partial O_{j}^{n}}{\partial a_{j}}=-\eta \delta_{j}\left(\frac{I_{j}^{p}-b_{j}}{a_{j}}\right) \\
& \Delta b_{j}=-\eta \frac{\partial E}{\partial b_{j}}=\eta \delta_{n} \omega_{j n} \frac{\partial O_{j}^{p}}{\partial b_{j}}=-\eta \delta_{j}
\end{aligned}
$$

The threshold variables $\Delta b 1_{j}$ and $\Delta b 2_{n}$ used in Eqs (40) and (41) are defined as follows:

$$
\begin{aligned}
& \Delta b 1_{j}=-\eta \frac{\partial E}{\partial b 1_{j}}=\eta \delta_{j} \\
& \Delta b 2_{n}=-\eta \frac{\partial E}{\partial b 2_{n}}=\eta \delta_{n}
\end{aligned}
$$

On the basis of the gradient descent algorithm, the weights, wavelet parameters and threshold values are updated and optimized through the following equations $[6,7]$ :

$$
\begin{aligned}
& \omega_{i j}(t+1)=\omega_{i j}(t)-\eta \frac{\partial E}{\partial \omega_{i j}}+\lambda \Delta \omega_{i j}(t) \\
& \omega_{j n}(t+1)=\omega_{j n}(t)-\eta \frac{\partial E}{\partial \omega_{j n}}+\lambda \Delta \omega_{j n}(t) \\
& a_{j}(t+1)=a_{j}(t)-\eta \frac{\partial E}{\partial a_{j}}+\lambda \Delta a_{j}(t) \\
& b_{j}(t+1)=b_{j}(t)-\eta \frac{\partial E}{\partial b_{j}}+\lambda \Delta b_{j}(t) \\
& b 1_{j}(t+1)=b 1_{j}(t)-\eta \frac{\partial E}{\partial b 1_{j}}+\lambda \Delta b 1_{j}(t) \\
& b 2_{n}(t+1)=b 2_{n}(t)-\eta \frac{\partial E}{\partial b 2_{n}}+\lambda \Delta b 2_{n}(t)
\end{aligned}
$$




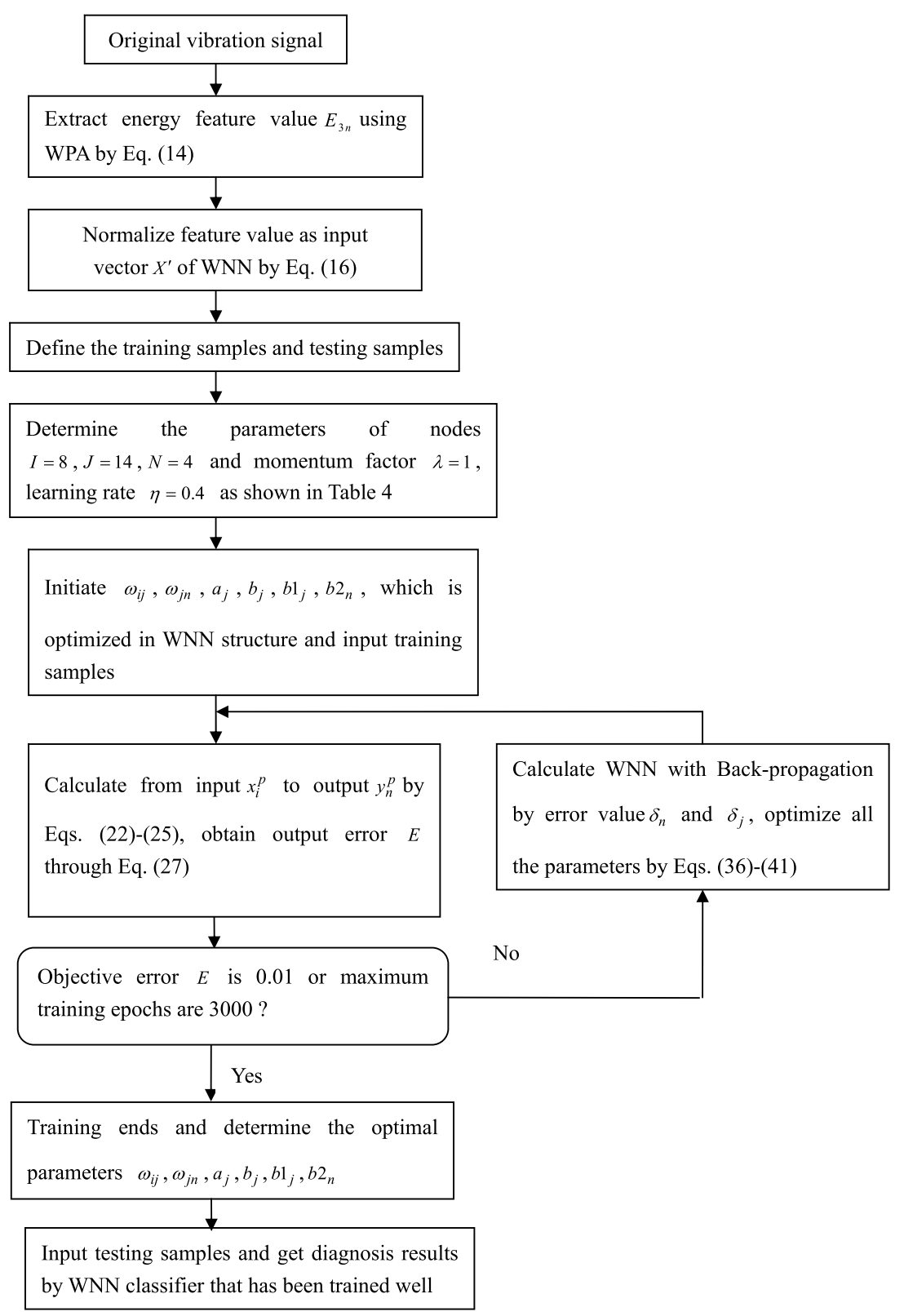

Fig. 3. The algorithm base on WPA for feature extraction and parameter optimization of WNN with BP algorithm.

\section{Fault detection of the gear cracks based on WPA and WNN}

The proposed method based on WPA and the wavelet neural network (WNN) with BP algorithm for the fault degradation detection of the gear crack in gearbox is shown in Fig. 3. The procedure including the extraction of the feature values from original vibration signals and the optimization of the wavelet parameters, weight coefficients and threshold values in WNN structure by BP algorithm are described as follows:

(1) Extract energy feature value $E_{3 n}$ of the original vibration signal collected from gearbox experimental system using WPA by Eq. (14).

(2) Normalize the extracted feature value by Eq. (16) and the vector $X^{\prime}$ is used as the input feature vector of WNN. 


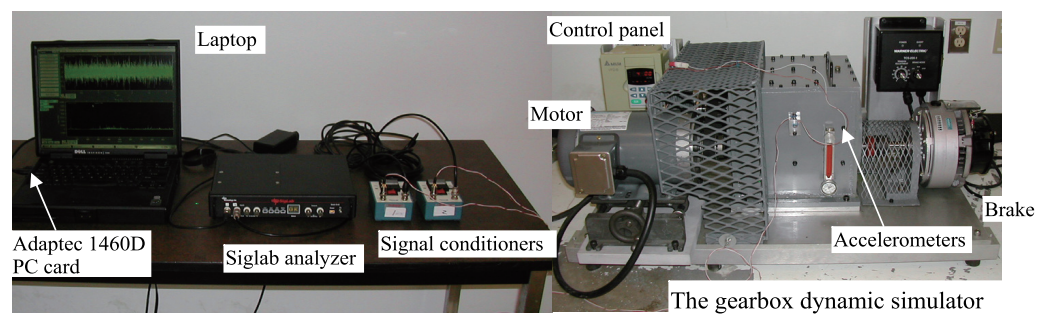

Fig. 4. The experimental apparatus of gearbox testing system.

(3) The extracted feature vectors are divided as the training samples and the testing samples.

(4) Determine the number of the nodes $I=8, J=14, N=4$, momentum factor $\lambda=1$, learning rate $\eta=0.4$, which is shown in Table 6 in order to obtain the best training results by the network.

(5) Initiate the weight coefficients $\omega_{i j}$ and $\omega_{j n}$, wavelet parameters $a_{j}$ and $b_{j}$, threshold values $b 1_{j}$ and $b 2_{n}$ in WNN structure and the input training samples.

(6) Train WNN based on Eqs (22)-(25) from the input $x_{i}^{p}$ of the input layer to the output $y_{n}^{p}$ of the output layer of WNN, the output error $E$ is obtained by comparing the actual output $y_{n}^{p}$ and the expected output $d_{n}^{p}$ by Eq. (27). If the output error $E$ does not meet the error requirement, turn to Step 7, otherwise, jump to Step 8. In this paper, the output error of the objective function in the network is 0.01 and the maximum training epochs are 3000 .

(7) Calculate WNN with the back-propagation algorithm. The back-propagation error values $\delta_{n}$ and $\delta_{j}$ are obtained by Eqs (28) and (29), optimize all the parameters of WNN from the output level to the input level based on the error signals using gradient descent algorithm. The weight coefficients $\omega_{i j}$ and $\omega_{j n}$ are optimized by Eqs (36) and (37). The wavelet parameters $a_{j}$ and $b_{j}$ are optimized by Eqs (38) and (39). The threshold values $b 1_{j}$ and $b 2_{n}$ are optimized by Eqs (40) and (41). After the optimization process is finished, it goes to Step 6.

(8) Check out the termination requirements. If the error $E$ in Eq. (27) meets the objective error setting or iterations achieve the maximum training epochs, thus training of network ends and determine all the parameters $\omega_{i j}$, $\omega_{j n}, a_{j}, b_{j}, b 1_{j}, b 2_{n}$.

(9) Input the testing samples and get diagnosis results by using WNN classifier, which is trained well by the above procedure.

\section{Gearbox experimental testing system}

The experimental apparatus of gearbox testing system is displayed in Fig. 4. This experimental apparatus consists of a gearbox, a dynamics simulator of SpectraQuest, two acceleration sensors of model 352C67PCB, a DSP Siglab 20-42 Signal Analyzer and a laptop. The gearbox dynamics simulator is used to collect the experimental vibration signals. The acceleration sensors are mounted on the gearbox in both vertical and horizontal directions. The vibration data are acquired by the Signal Analyzer and laptop with the data acquisition software.

Figure 5 shows the experimental system of the gearbox, which is used for the fault degradation detection of the crack sizes of the gear. The sensors mounted in a vertical and horizontal direction acquire the experimental vibration signal. One of the gears 3 and 4 is chosen to simulate the faults in this experiment. Tooth meshing of gears 3 and 4 is considered as the dynamic load on a cantilever beam. The deflection has close relation with the force applied to the end of the beam, the length of the beam, Young Modulus of the material and the inertia moment of the beam. Z1 is the rotational speed of shaft 1 and gear 1, Z2 is the rotational speed of shaft 2 and gears 2 and 3, Z3 is the rotational speed of shaft 3 and gear 4, Z12 is the meshing frequency of gears 1 and 2, Z34 is the meshing frequency of gears 3 and 4.

The purpose of the experiment is to test the proposed methods of fault diagnosis and develop the relation between faults and signatures. Figure 6 shows the critical area of the gear.

Here the parameter $a$ is one half of the chordal tooth thickness. The parameter $b$ is the face width of the tooth with $25 \mathrm{~mm}$. The crack widths of the fault modes F2, F3, and F4 are about $25 \%, 50 \%$ and $75 \%$ of the face width (b), 


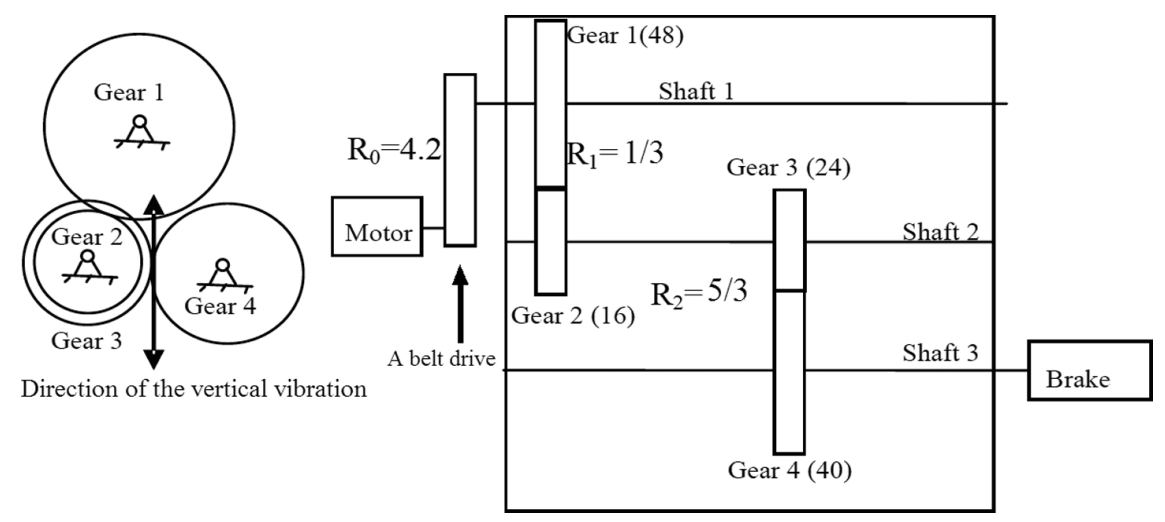

Fig. 5. The diagram of the experimental system.

Table 2

The geometry parameters of the test gear

\begin{tabular}{lc}
\hline Parameter & Gear 3 \\
\hline Type & MA25-24S \\
Number of teeth & 24 \\
Module & 2.5 \\
Face width (mm) & 25 \\
Pressure angle (deg) & 20 \\
Helix angle (deg) & 0 \\
Pitch diameter (mm) & 50 \\
Material (mild steel) & EN8 \\
\hline
\end{tabular}

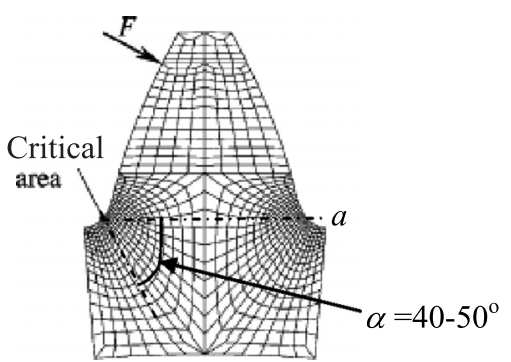

Fig. 6. The critical area of the tooth of the gear in gearbox.

Table 3

Geometry of the crack fault in our experiment

\begin{tabular}{ccccc}
\hline \multirow{2}{*}{ Crack fault modes } & \multicolumn{4}{c}{ Geometry of fault } \\
\cline { 2 - 5 } & Depth $(\mathrm{mm})$ & Width $(\mathrm{mm})$ & Thickness $(\mathrm{mm})$ & Angle (degree) \\
\hline F2 & $(1 / 4) \mathrm{a}$ & $(1 / 4) \mathrm{b}$ & 0.4 & \\
F3 & $(1 / 2) \mathrm{a}$ & $(1 / 2) \mathrm{b}$ & 0.4 & $45^{\circ}$ \\
F4 & $(3 / 4) \mathrm{a}$ & $(3 / 4) \mathrm{b}$ & 0.4 & \\
\hline
\end{tabular}

Table 4

Four gear fault modes

\begin{tabular}{clrlll}
\hline Fault modes & Fault description & \multicolumn{4}{c}{ Target output modes } \\
\hline F1 & Cracks at levels of 0\% & 1 & 0 & 0 & 0 \\
F2 & Cracks at levels of 25\% & 0 & 1 & 0 & 0 \\
F3 & Cracks at levels of 50\% & 0 & 0 & 1 & 0 \\
F4 & Cracks at levels of 75\% & 0 & 0 & 0 & 1 \\
\hline
\end{tabular}

respectively. The crack depths of the fault modes F2, F3, and F4 are about 25\%, $50 \%$ and $75 \%$ of the parameter a. The crack thickness of the gear 3 is $0.4 \mathrm{~mm}$, which is determined by the thickness of the cutting tool. The geometry parameters of the tested Gear 3 are listed in Table 2 and the geometry of the crack fault in our experiment is shown in Table 3. And the crack in gear is generated along the normal line of the tooth's root curve with an angle of 45. The four gear fault modes and the target output modes of WNN are defined in Table 4 to test the proposed method's effectiveness for the identification of the fault category with the different gear damage levels. The selected rotational speed and characteristic frequency of gearbox are shown in Table 5. When the rotational speed is constant, there are two types of loads, which is half load and full load. The full load under each motor speed is shown in Table 5.

In Ref [11,12], the weighted K nearest neighbor classification algorithm and a multidimensional hybrid intelligent method were proposed to diagnose the three fault modes under F1, F2 and F3 under the different rotational speeds and the load torques. In Ref [12], a multidimensional hybrid method for the fault gear diagnosis was proposed. The 
Table 5

Rotational speed and characteristic frequency of the gearbox

\begin{tabular}{cccrrrr}
\hline Motor rpm & Torque (N.m) less than & Z1(Hz) & Z12(Hz) & Z2(Hz) & Z34(Hz) & Z3(Hz) \\
\hline 800 & 51.77 & 3.17 & 152.38 & 9.52 & 228.57 & 5.71 \\
1200 & 34.51 & 4.76 & 228.57 & 14.29 & 342.86 & 8.57 \\
1600 & 25.89 & 6.35 & 304.76 & 19.05 & 457.14 & 11.43 \\
\hline
\end{tabular}

Table 6

The selected nodes, momentum term and learning rate of WNN

$\begin{array}{lc}\text { Nodes of input layer } & I=8 \\ \text { Nodes of hidden layer } & J=14 \\ \text { Nodes of output layer } & N=4 \\ \text { Momentum term } & \lambda=1 \\ \text { Learning rate } & \eta=0.4\end{array}$

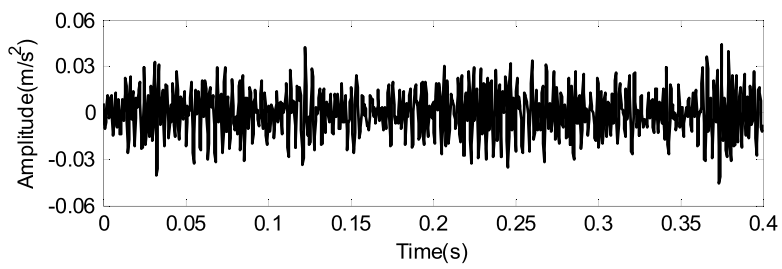

(a)

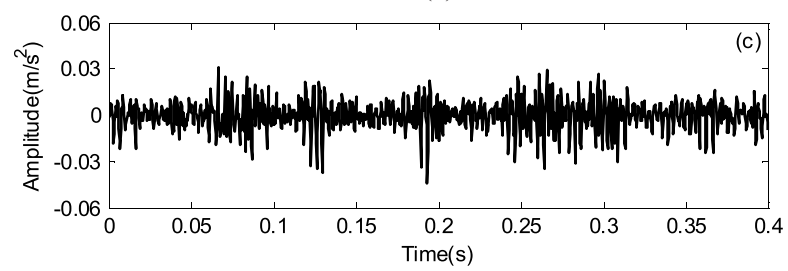

(c)

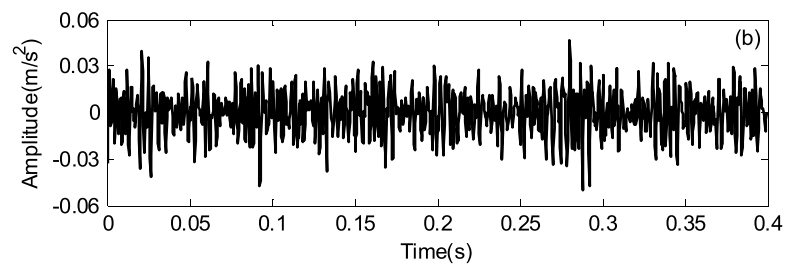

(b)

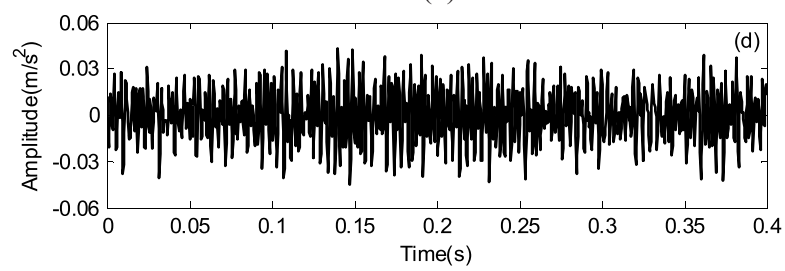

(d)

Fig. 7. The experimental vibration signal of the gearbox under four conditions, (a) $F_{1}$, (b) $F_{2}$, (c) $F_{3}$ and (d) $F_{4}$.

fault modes of the gear are the chipped tooth and missing tooth, which is more obvious and easier to be diagnosed than that in the experiment in this paper. Meanwhile, there are three fault modes of the gear cracks in gearbox to be diagnosed in Refs [11-21]. In this paper, we proposed the novel method to diagnose the four fault modes under F1, F2, F3 and F4 under the different rotational speeds and load torques, which is one more fault modes than that in Refs $[11,12]$. The classification success rate under F1, F2 and F3 by the proposed method is much better than that in Refs [11,12].

\section{Results and discussions}

As discussed above, the experimental vibration signals are collected from the gearbox system under four gear conditions. Figure 7 shows the experimental vibration signal under $\mathrm{F}_{1}, \mathrm{~F}_{2}, \mathrm{~F}_{3}$ and $\mathrm{F}_{4}$ conditions when the rotational speed is $800 \mathrm{rpm}$ and the load torque is full load. The vibration signal in time domain does not present any information, which is used to detect the fault condition of the gears in gearbox. In order to detect the fault degradation of the gears in gearbox with the feature values in time domain, the method on feature extraction based on WPA is proposed, which is introduced in Section 2. The three-level decomposition of WPA for the vibration signal analysis is introduced. Figure 8 shows the three-level decomposition of the vibration signal under $\mathrm{F}_{2}$ fault condition when the rotational speed is $800 \mathrm{rpm}$ and the load torque is full load. WPA decomposes the experimental vibration signal within the eight frequency bandwidths. Figure 9 shows the reconstructed vibration signal of $(3,1)$ in three-level decomposition of WPA under $\mathrm{F}_{1}, \mathrm{~F}_{2}, \mathrm{~F}_{3}$ and $\mathrm{F}_{4}$ gear conditions. The difference of the energy in time domain is slightly 

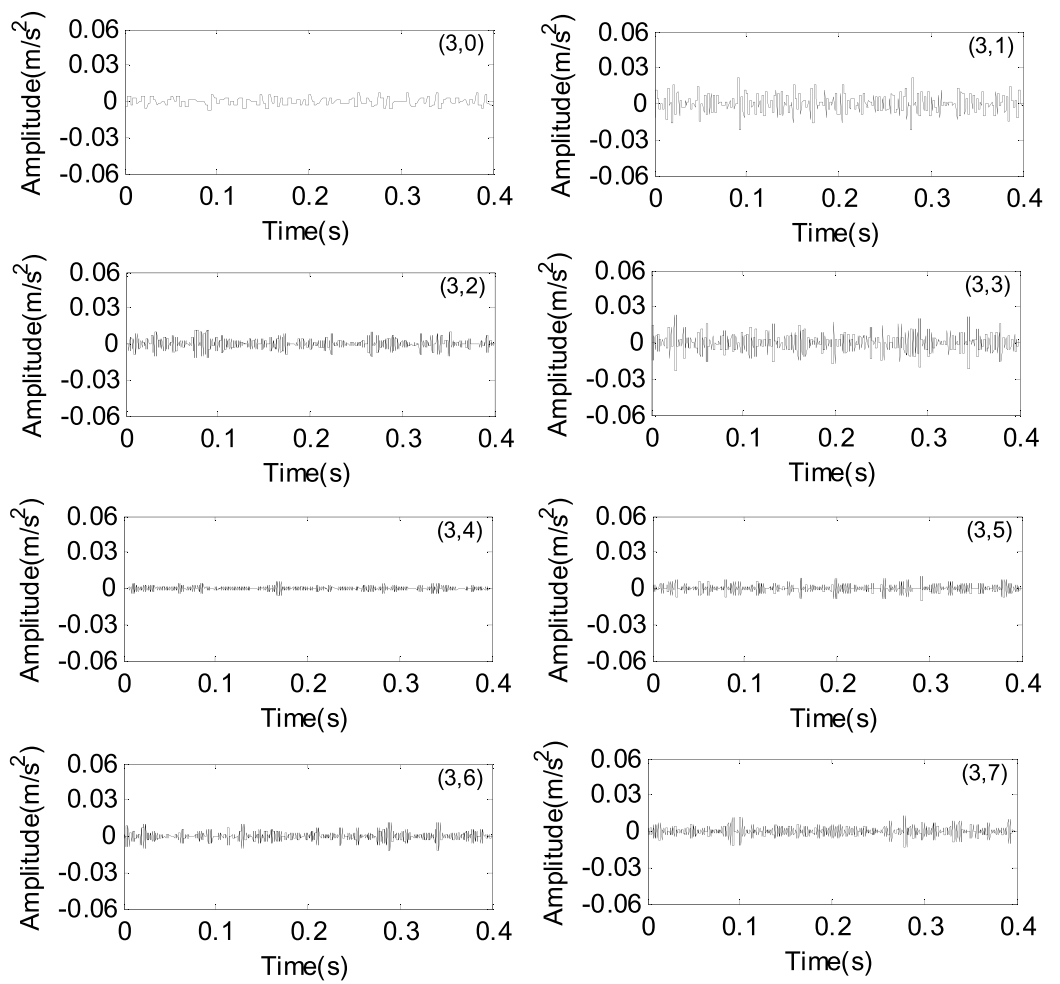

Fig. 8. Three-level decomposition of the experimental vibration signal under $\mathrm{F}_{2}$ fault condition.

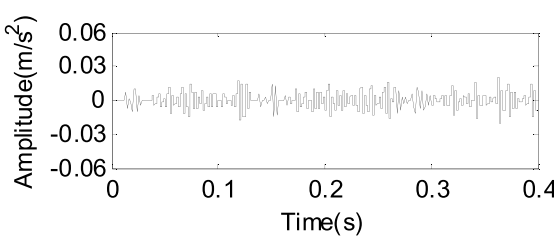

(a)

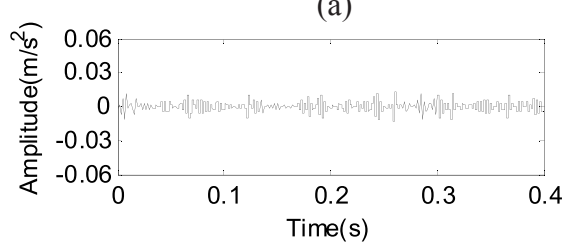

(c)

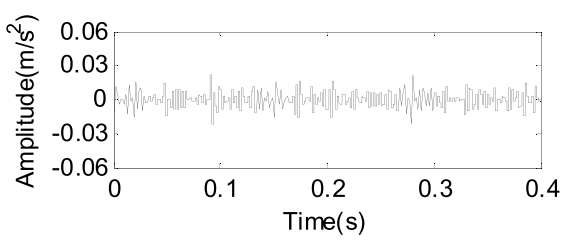

(b)

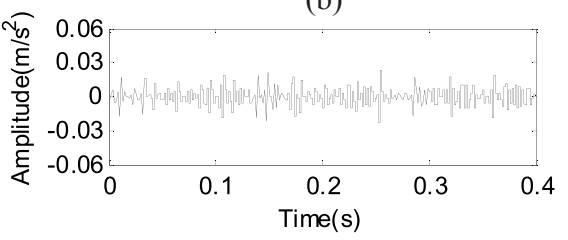

(d)

Fig. 9. The reconstructed vibration signal of $(3,1)$ in three-level decomposition of WPA under four conditions, (a) $F_{1}$, (b) $F_{2}$, (c) $F_{3}$ and (d) $F_{4}$.

different, which is not enough to classify the fault conditions of the gears in gearbox. As described in Table 1 and Eqs (15)-(16), the energy of the reconstructed vibration signals in three-level decomposition of WPA is calculated and used as the input feature values of WNN to detect the fault degradation of the gear cracks in gearbox.

Figure 10(a) shows that the energy under the $\mathrm{F}_{4}$ fault condition is larger than that under the other three conditions. Figures $10(\mathrm{~b})-(\mathrm{h})$ show that the energy under $\mathrm{F}_{3}$ is lower than that under other three conditions. It is not enough to make decision on the gear conditions in gearbox by the visual images as shown in Fig. 10. It is necessary to develop the intelligent methodology to detect the fault degradation of the gear cracks in gearbox by using the data in Fig. 10 . As proposed procedure in Section 4, the method based on WNN is applied to identify the gear conditions in gearbox.

The effectiveness for the detection of the gear cracks by WNN has relation with the parameters in WNN. It 


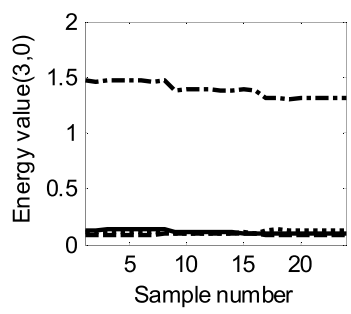

(a)

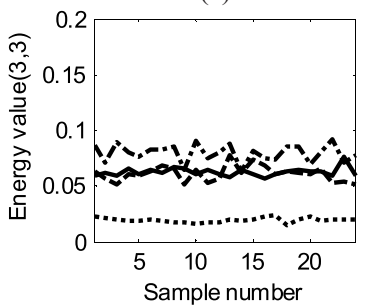

(d)

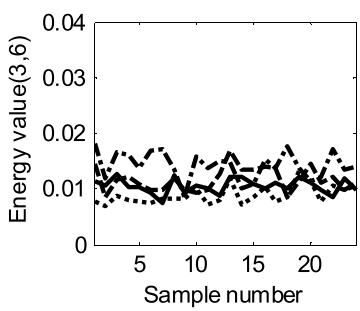

(g)

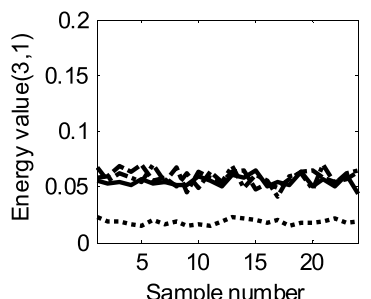

(b)

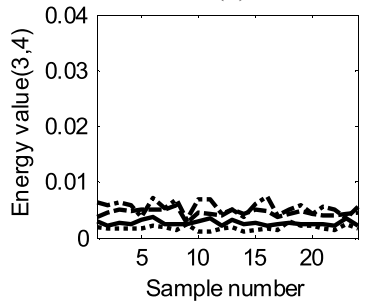

(e)

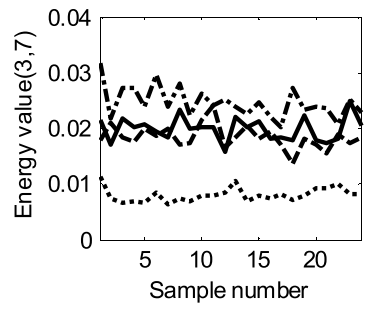

(h)

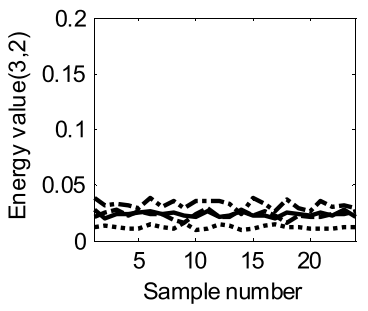

(c)

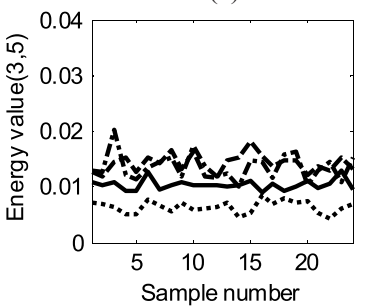

(f)

Fig. 10. The energy feature values of the eight reconstructed vibration signals in three-level decomposition of WPA under $\mathrm{F}_{1}$ (solid), $\mathrm{F}_{2}$ (dash), $\mathrm{F}_{3}$ (dot) and $\mathrm{F}_{4}$ (dash-dot) gear condition.

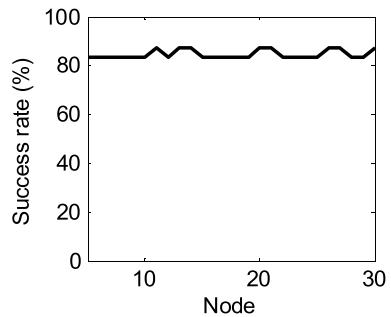

(a)

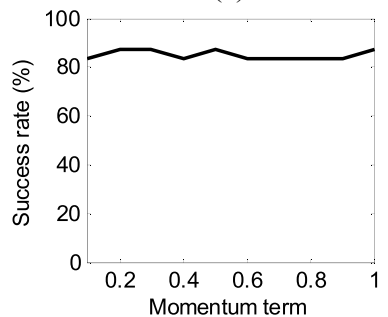

(c)

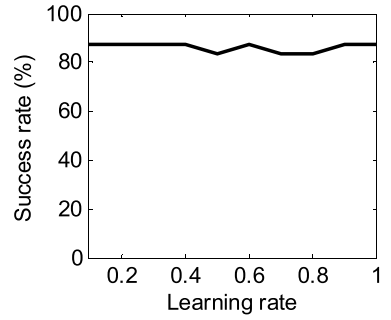

(b)

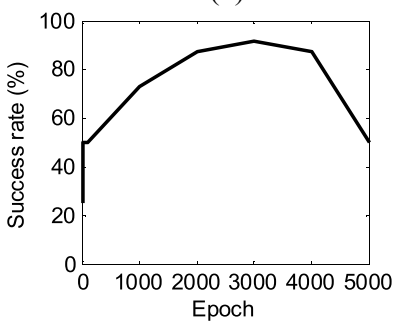

(d)

Fig. 11. The effects of the parameters in WNN on the success rate: (a) Node of WNN, (b) Momentum term, (c) Learning rate, and (d) Epoch.

is necessary to optimize the parameters in WNN structure, including the number of nodes of the hidden layer, momentum factor, learning rate. Figure 11 shows effects of the four important parameters on the average success rate of the classification under four gear conditions in gearbox. Figure 11(a) shows the relation between number of 
Table 7

The classification success rate with the vertical vibration signal analysis

\begin{tabular}{|c|c|c|c|c|c|c|}
\hline & \multicolumn{2}{|c|}{$800 \mathrm{r} / \mathrm{min}$} & \multicolumn{2}{|c|}{$1200 \mathrm{r} / \mathrm{min}$} & \multicolumn{2}{|c|}{$1600 \mathrm{r} / \mathrm{min}$} \\
\hline & Half load & Full load & Half load & Full load & Half load & Full load \\
\hline F1 (\%) & 83.33 & 100 & 75 & 75 & 100 & 91.67 \\
\hline $\mathrm{F} 2(\%)$ & 91.67 & 91.67 & 100 & 91.67 & 100 & 100 \\
\hline F3 (\%) & 100 & 100 & 91.67 & 100 & 100 & 100 \\
\hline $\mathrm{F} 4(\%)$ & 100 & 100 & 100 & 100 & 100 & 100 \\
\hline
\end{tabular}

Table 8

The classification success rate with the horizontal vibration signal analysis

\begin{tabular}{|c|c|c|c|c|c|c|}
\hline & \multicolumn{2}{|c|}{$800 \mathrm{r} / \mathrm{min}$} & \multicolumn{2}{|c|}{$1200 \mathrm{r} / \mathrm{min}$} & \multicolumn{2}{|c|}{$1600 \mathrm{r} / \mathrm{min}$} \\
\hline & Half load & Full load & Half load & Full load & Half load & Full load \\
\hline F1 (\%) & 100 & 100 & 100 & 100 & 100 & 100 \\
\hline F2 (\%) & 100 & 100 & 100 & 100 & 100 & 100 \\
\hline F3 (\%) & 100 & 100 & 100 & 100 & 100 & 100 \\
\hline F4 (\%) & 100 & 100 & 100 & 100 & 100 & 100 \\
\hline
\end{tabular}

nodes of the hidden layer and success rate when the rotational speed is $800 \mathrm{rpm}$ and the load torque is full load. When the number of nodes of the hidden layer is small, the success rate is not good enough. The system output error of the training network is reduced when the number of nodes of the hidden layer is large, which produce the prolonged training time of network and the results of the training WNN is easy to be the local minimum. The principle of selecting the number of nodes of hidden layer of network is that structure is compact enough both to meet the requirement of the accuracy and choose small number of nodes of hidden layer. Here the number of nodes of the hidden layer is 14. Figure 11(b) shows the relation between the success rate and the learning rate. The learning rate determines the stability of the learning process of WNN. The large learning rate is difficult to make the WNN network to converge to the certain minimum value. The small learning rate results in the long learning time of WNN network. Based on the convergence and stability of WNN network, the learning rate between 0.01 and 0.8 is much better. The learning rate is chosen to be 0.4 . Figure 11(c) shows the relation between the success rate and momentum term. The increasing momentum term is used to avoid the local minimum points of the training of network. Theoretically, the momentum term is usually between 0 and 1 and is larger than the learning rate. The momentum coefficient of the WNN network is selected to be 1 . The number of nodes of input layer of network is $I=8$, which corresponds the eight feature input parameters. The number of nodes of the output layer is $N=4$ for the four fault modes. The number of nodes of WNN, momentum term and learning rate are shown in Table 6.

As described in Fig. 3, the proposed method is applied to analyze the vertical and horizontal vibration signals. There are twenty-four set of the experimental vibration signals along the horizontal and vertical directions, which are collected from the gearbox system. The twelve set of the feature values are used as the training data to WNN and twelve set of feature value are used as the testing data to WNN. Table 7 shows the classification success rate of the four gear conditions by analyzing the vertical vibration signal under the different load torques and rotational speeds. When the rotational speed is $1200 \mathrm{rpm}$ and the load is half and full, the classification success rate under $F_{1}$ is $75 \%$, which are not good enough by comparison with other success rates. When the rotational speed increased to be $1600 \mathrm{rpm}$, the success rates under half load and full load is $100 \%$ and $91.67 \%$, which is higher than that with the rotational speed of $1200 \mathrm{rpm}$. The other success rates in Table 7 are more than $90 \%$. Table 8 shows the success rate of the classification by the horizontal vibration signal. All the success rates is $100 \%$ under different loads and rotational speeds. The results on the horizontal vibration signal analysis in Table 8 are much better than that for the vertical vibration signal analysis in Table 7 . The horizontal vibration signal is more sensitive to the development of the gear crack in gearbox than the vertical vibration signal. Based on the above analysis results, the proposed method in the paper is effective and reliable for the fault degradation of the gear cracks in gearbox under the different torques and rotational speeds.

The effectiveness and robustness for the fault detection of the gear cracks in gearbox by the proposed method in this paper is tested by the comparison with the results in Ref [11,12]. In Refs [11,12], the proposed method just can detect and classify the three fault modes about F1, F2 and F3. Especially, the range of the accuracy of the classification in Ref [12] is from $86.11 \%$ to $100 \%$. The average classification rates under the different training and 
testing mode is $98.33 \%$. In this paper, the proposed method can diagnose and classify the four fault modes with F1, F2, F3 and F4, which is more than three fault modes with F1, F2 and F3 in Refs [11,12]. As shown in Table 8, all of the classification rates for the horizontal vibration signals are $100 \%$ and the average classification rate is $100 \%$, which are higher than the results in Ref [12].

In Table 7, the classification success rates for the vertical vibration signals are between $83.33 \%$ and $100 \%$ except two classification success rates for F1 under the rotational speed of $1200 \mathrm{r} / \mathrm{min}$. The average classification rate except the $75 \%$ under F1 and including the $75 \%$ under F1 is $88.6 \% 87 \%$, respectively. The $75 \%$ classification success rates under F1 in Table 7 are less than that in Refs [11,12]. The important reason for $75 \%$ classification rate is that the classification rate by WNN has close relationship with the inputted feature values by WPA. The sets of the feature values under four types of the fault modes are more complicated than that under three types of the fault modes in Refs $[11,12]$. One more fault mode with F4 in this paper increases the complexity and difficulties in the feature extraction by WPA and the classification of the multiple fault modes of gearbox by the WNN, which reduces the classification success rate. Meanwhile, it tests the robustness of the proposed method based on WPA and WNN.

The classification success rates for the horizontal vibration signal are 100\%, which is higher than that for the vertical vibration signal. It shows the horizontal vibration signal is sensitive to the gear crack than the vertical vibration signal. Because the inputted feature values in WNN under the different fault conditions have close relation with the dynamic structure of the gearbox system, which is shown Fig. 5.

The proposed method in this paper combines the advantages of the methods with WPA to extract the feature values and WNN as the classification method, which is effective and robust for the fault degradation identification of gearbox.

\section{Conclusion}

This paper proposed a novel method based on wavelet neural network to detect the different fault modes with the four gear crack sizes. The wavelet packet analysis is used to preprocess the vibration signal to extract feature values of the vibration signals in time domain under different loads and rotational speeds. The algorithm for the fault detection of the gear cracks based on wavelet neural network is proposed. The optimization for the parameters in WNN is introduced. The experimental gearbox system is designed to collect the horizontal and vertical vibration signal under different loads and rotational speeds for the different fault modes of the gearbox. The results show that the proposed method is effective to identify the different damage levels and categories of the gear cracks under multiple fault modes.

\section{Acknowledgment}

The experimental data is provided by the Reliability Research Lab in the Department of Mechanical Engineering at the University of Alberta in Canada. The project is supported by the National Natural Science Foundation of China (61273176), Program for New Century Excellent Talents in University of Minister of Education of China (201010621237), Major project of Hubei Provincial Department of Education (Z20101501) and scientific and technological project of Wuhan Science and Technology Bureau (201010621237).

\section{References}

[1] H. Zheng, Z. Li and X. Chen, Gear fault diagnosis based on continuous wavelet transform, Mechanical Systems and Signal Processing 16 (2002), 447-457.

[2] H.Y. Yang, J. Mathew and L. Ma, Intelligent diagnosis of rotating machinery faults-A review, 3rd Asia-Pacific Conference on Systems Integrity and Maintenance, Cairns, Australia, 2002.

[3] H.X. Chen, P.S.K. Chua and G.H. Lim, Feature extraction optimization and classification by second generation wavelet and support vector machine for fault diagnosis of water hydraulic power system, International Journal of Fluid Power 7 (2006), 39-52.

[4] J. Rafiee, F. Arvani, A. Harifi and M.H. Sadeghi, Intelligent condition monitoring of a gearbox using artificial neural network, Mechanical Systems and Signal Processing 21 (2007), 1746-1754. 
[5] Q. Zhang and A. Benveniste, Wavelet networks, IEEE Transactions on Neural Networks 3 (1992), 889-898,

[6] Y.H. Chen, B. Yang and J.W. Dong, Time-series prediction using a local linear wavelet neural network, Neurocomputing 69 (2006), 449-465.

[7] N. Chauhan, V. Ravi and D.K. Chandra, Differential evolution trained wavelet neural network: Application to bankruptcy prediction in banks, Expert Systems with Applications 36 (2009), 7659-7665.

[8] H.R. Berenji and Y. Wang, Wavelet neural networks for fault diagnosis and prognosis, IEEE 14th International Conference on Fuzzy Systems, Vancouver, Canada, (2006), 1334-1339.

[9] Y. Kang, C.C. Wang and Y.P. Chang, Gear fault diagnosis by using wavelet neural networks, Advances in Neural Networks 4493 (2007), $580-588$.

[10] Q. Huang, D.X. Jiang, L.Y. Hong and Y.S. Ding, Application of wavelet neural networks on vibration fault diagnosis for wind turbine gearbox, Advances in Neural Networks 5264 (2008), 313-320.

[11] Y.G. Lei and M.J. Zuo, Gear crack level identification based on weighted K nearest neighbor classification algorithm, Mechanical Systems and Signal Processing 23 (2009), 1535-1547.

[12] Y.G. Lei, M.J. Zuo, Z.J. He and Y.Y. Zi, A multidimensional hybrid intelligent method for gear fault diagnosis, Expert Systems with Applications 37 (2010), 1419-1430.

[13] H.X. Chen, P.S.K. Chua and G.H. Lim, Fault degradation assessment of water hydraulic motor by impulse vibration signal with wavelet packet analysis and kolmogorov-smirnov test, Mechanical Systems and Signal Processing 22 (2008), 1670-1684.

[14] X.F. Fan and M.J. Zuo, Gearbox fault detection using hilbert and wavelet packet transform, Mechanical Systems and Signal Processing 20 (2006), 966-982

[15] B. Liu, Selection of wavelet packet basis for rotating machinery fault diagnosis, Journal of Sound and Vibration 284 (2005), 567-582.

[16] C. Pan, W. Chen and Y. Yun, Fault diagnostic method of power transformers based on hybrid genetic algorithm evolving wavelet neural network, IET Electric Power Applications 2 (2008), 71-76. 

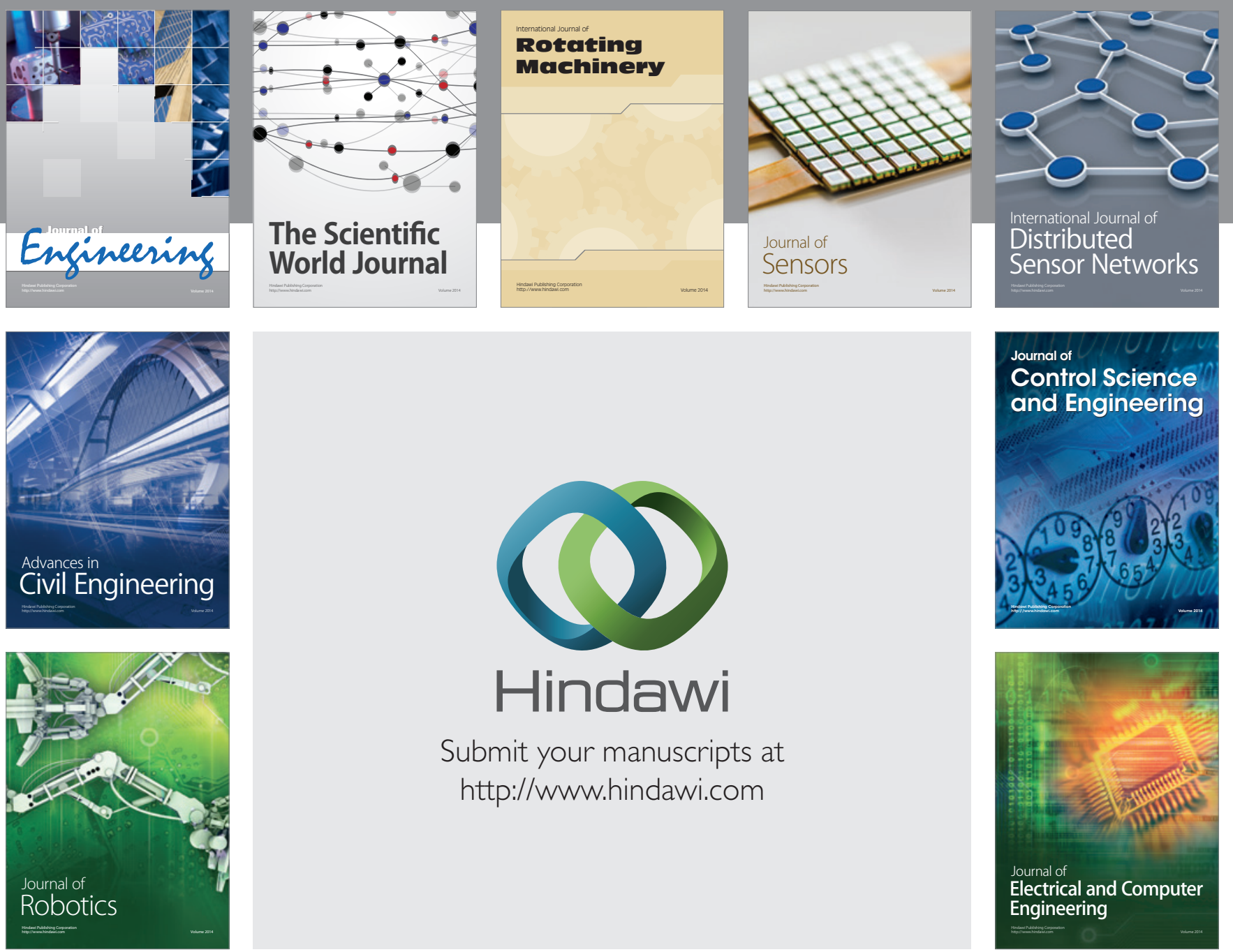

Submit your manuscripts at

http://www.hindawi.com
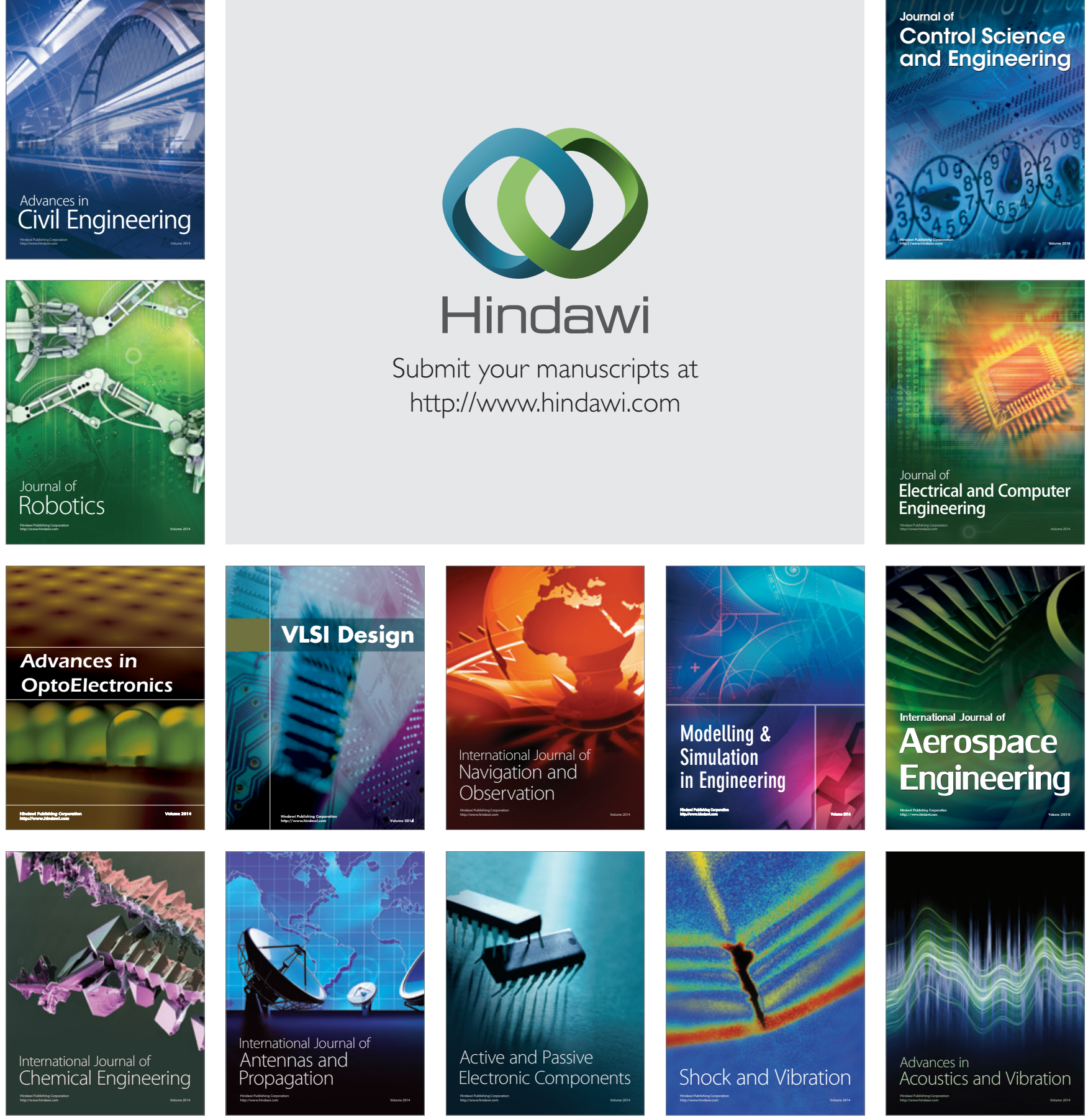